\title{
Pars Triangularis Volume Asymmetry and Schneiderian First Rank Symptoms in Antipsychotic-naïve Schizophrenia
}

\author{
Venkataram Shivakumar ${ }^{1, *}$, Vanteemar Sathyanarayana Sreeraj ${ }^{2, *}$, Sunil Vasu Kalmady ${ }^{3}$, \\ Bangalore Nanjundaiah Gangadhar ${ }^{2}$, Ganesan Venkatasubramanian² \\ Departments of ${ }^{1}$ Integrative Medicine and ${ }^{2}$ Psychiatry, National Institute of Mental Health \& NeuroSciences (NIMHANS), Bangalore, India, \\ ${ }^{3}$ Alberta Machine Intelligence Institute, University of Alberta, Edmonton, AB, Canada
}

\begin{abstract}
Objective: Schizophrenia is a disorder of language and self, with first-rank symptoms (FRS) as one of the predominant features in a subset of patients. Abnormal language lateralization is hypothesized to underlie the neurobiology of FRS in schizophrenia. The role of Broca's area with its right-hemispheric counterpart, consisting of pars triangularis (PTr) and pars opercularis (POp) of the inferior frontal gyrus in FRS is undetermined. We compared the volumes and asymmetries of PTr \& POp in anti-psychotic-naive schizophrenia patients with FRS (FRS[+]) with those without FRS (FRS[-]) and healthy-controls $(\mathrm{HC})$ using three dimensional, interactive, semi-automated volumetric morphometry.

Methods: Antipsychotic naïve FRS $(+)(n=27)$, FRS $(-)(n=24)$ and HC $(n=51)$ were carefully assessed with structured and semi-structured clinical tools. T1-weighted images were acquired in a 3T scanner. Volumes of regions of interest were measured independently for both sides using slicer-3D software, and asymmetry indices were calculated.

Results: FRS(+) but not FRS $(-)$ had a significant volume deficit in right PTr after controlling for the potential confounding effects of age, sex, and intracranial volume $(p=0.029)$. There was a significant leftward asymmetry of PTr in patients with FRS (i.e., leftward asymmetry in patients) $(p=0.026)$. No significant volume/asymmetry abnormalities were observed in POp.

Conclusion: Study findings suggest reduced right PTr volume with leftward asymmetry to be associated with FRS in schizophrenia. This is consistent with the loss of Yakovlevian torque in schizophrenia. Role of PTr in the neurobiology of schizophrenia as a disorder of self, speech, and social cognition needs further systematic evaluation in future research.
\end{abstract}

KEY WORDS: Schizophrenia; Broca area; Language; Schneiderian first-rank symptoms.

\section{INTRODUCTION}

Language acquisition by humans has been hypothesized to be one of the vital evolutionary factors that might have culminated in schizophrenia [1,2]. Indeed, language dysfunction has been considered to be critical in schizophrenia pathogenesis - perhaps the most important - as it has been reported to differentiate schizophrenia from other psychoses. It is known that patients with schizophrenia find it difficult to process semantic aspects of language ap-

Received: July 20, 2020 / Revised: December 14, 2020 Accepted: February 2, 2021

Address for correspondence: Ganesan Venkatasubramanian Department of Psychiatry, National Institute of Mental Health \& NeuroSciences (NIMHANS), Hosur Road, Bengaluru 560029, India E-mail: gvs@nimhans.ac.in

ORCID: https://orcid.org/0000-0002-0949-898X

*These authors contributed equally to this study. propriately [3], their speech is 'disorganized' [4] and they demonstrate reduced comprehension accuracy according to syntax [5].

Language is a function of the speech areas of the brain. Inferior frontal gyrus (IFG), which comprises Broca's area and its right counterpart, is one of the critical speech areas of the brain. IFG consists of pars triangularis (PTr) (Brodmann's area [BA] 44) and pars opercularis (POp) (Brodmann's area [BA] 45) that are anatomically and functionally heterogeneous [6]. Various functional and anatomical studies have implicated the role of IFG in language processing [7] and previous studies have suggested an essential role of IFG abnormality in schizophrenia [8]. Various imaging studies, both manual and automated, have demonstrated a significant relationship between symptom severity and reduced IFG volume in schizophrenia [9].

(c) This is an Open-Access article distributed under the terms of the Creative Commons Attribution Non-Commercial License (http://creativecommons.org/licenses/by-nc/4.0) which permits unrestricted non-commercial use, distribution, and reproduction in any medium, provided the original work is properly cited. 
Closely associated with language dysfunction is the hypothesis that people who suffer from schizophrenia display abnormal lateralization of language. The concept of asymmetry being the defining feature of the human brain was emphasized by the assertion of Paul Broca that: "Man is, of all the animals, the one whose brain is most asymmetrical" [10]. Interestingly, several structural and functional studies suggest that schizophrenia is associated with reduced lateralization of language to the left hemisphere; some even report a reversal of lateralization, to the right hemisphere [11-14]. Thus, in patients with schizophrenia, the right hemisphere's apparent involvement in the mediation of left hemisphere language functions is of considerable significance. But these studies have examined planum temporale (Wernicke's area) primarily, and there is a dearth of knowledge on the structural asymmetry of Broca's area in health and disease [15].

Language lateralization is hypothesized to be of further importance in the manifestation of Schneiderian first-rank symptoms (FRS) [16,17]. Though nosological systems have downgraded the importance of FRS in diagnosing schizophrenia, the possibility of subgroups with FRS having distinct neurobiological underpinning cannot be discounted for $[18,19]$. A distinctive phenotype of the disrupted boundary of self may underlie the FRS [20] and abnormal lateralization with dysfunctional language areas would have a central role in its pathogenesis $[21,22]$. Aberrations in lateralization can explain the language deficits in schizophrenia; specifically, in schizophrenia patients, the components of language that are typically segregated to the left hemisphere are less strongly lateralized $[16,17]$. The right dorsolateral prefrontal cortex facilitates the transition from engrams representing the precursors of speech ('thoughts' and 'intentions') to the linear phonological output that is assembled in and around Broca's area in the dominant hemisphere $[16,17]$. Hence, the FRS like thought insertion and withdrawal has been proposed to result from activation or deactivation of typically autonomous 'thoughts' and 'intentions' in the right dorsolateral prefrontal cortex (especially involving the Broca's area) by incoming sensory stimuli $[16,17]$. Studies that examine the neuroanatomical features of Broca's area like volume and asymmetry in schizophrenia patients with and without FRS might help us to understand the neuroanatomical basis of these symptoms. Hence, in this study, we examined the volume and symmetry of PTr and POp in anti- psychotic-naive schizophrenia patients with FRS (FRS $[+])(n=27)$ in comparison to those without FRS (FRS $[-])(n=24)$ and healthy control $(\mathrm{HC})(n=52)$ using a three dimensional, interactive, semi-automated analysis with good inter-rater reliability.

\section{METHODS}

Patients attending the clinical services of the tertiary psychiatric care center, who fulfilled Diagnostic and Statistical Manual of Mental Disorders 4th edition criteria for schizophrenia and were never treated with any psychotropic medications including antipsychotics \& not having substance abuse ( $n=51$; M:F $=25: 26$ ), were examined in this study. The diagnosis of schizophrenia was established using Mini International Neuropsychiatric Interview Plus (MINI) [23], which was confirmed by another psychiatrist through an independent clinical interview. FRS were examined using a comprehensive semistructured interview as per the established descriptions [24]; these 11 symptoms described by Mellor included the following: audible thoughts, voices arguing, voices commenting, thought insertion, thought withdrawal, thought broadcast, made feelings, made impulses, made volitions, somatic passivity and delusional percept [24]. Besides, the presence of FRS was independently ascertained by two qualified psychiatrists by a comprehensive mental status examination. Depending on the presence of at least one FRS, schizophrenia patients were classified into FRS(+) and FRS( - ). Psychopathology was assessed using Scales for the Assessment of Positive and Negative Symptoms $[25,26]$. The illness characteristics and antipsychotic-naïve status were carefully ascertained by reliable information obtained from at least one first-degree relative.

$H C(n=52 ; M: F=1: 1)$, who volunteered for the study, were screened to rule out any psychiatric diagnosis using the MINI as well as a comprehensive mental status examination. None of the $\mathrm{HC}$ had a family history of psychiatric disorder in first-degree relatives. Patients and $\mathrm{HC}$ did not have features suggestive of alcohol abuse/ dependence. None used stimulant or opiate drugs. None had a history or any clinical feature suggestive of neurological/medical disorder. All the subjects were right-handed (established using Edinburgh handedness inventory). After a complete description of the study to the subjects, informed consent 
was obtained. The Institute's ethics committee approved the study (IEC no: No. NIMHANS/74th IEC/2011 dated 03 May 2011).

\section{Scanning Protocol}

Magnetic resonance imaging was done with Philips Achieva 3Tesla scanner (Philips Medical Systems, Best, Netherlands). T1 weighted images were acquired using the following parameters, Repetition time $(T R)=8.1 \mathrm{msec}$, Echo time $(\mathrm{TE})=3.7 \mathrm{msec}$, nutation angle $=8$ degrees, Field-of-View $($ FoV $)=256 \mathrm{~mm}$, slice thickness $=1 \mathrm{~mm}$ without inter-slice gap, Number of excitations $($ NEX) $=1$, matrix $=256 \times 256$. The digital image and communication on medicine (DICOM) images were transferred to a personal computer and stored with coded identification.

\section{Regions of Interest (Rol)}

In this study, the region of interest, the IFG comprising of PTr and POp on both left and right sides, was measured using the software 'Slicer 3D' (www.slicer.org). The region of interest was traced manually using a mouse-driven computer-guided pointer following strict anatomical guidelines [27]. The Rol was traced on sagittal sections, as depicted in Figure 1. The PTr extended superiorly to the inferior frontal sulcus, inferiorly to the anterior horizontal ramus, and caudally to the anterior ascending ramus (AAR). The POp is located adjacent to the PTr, posteriorly. The POp is bounded superiorly by the inferior frontal sul-

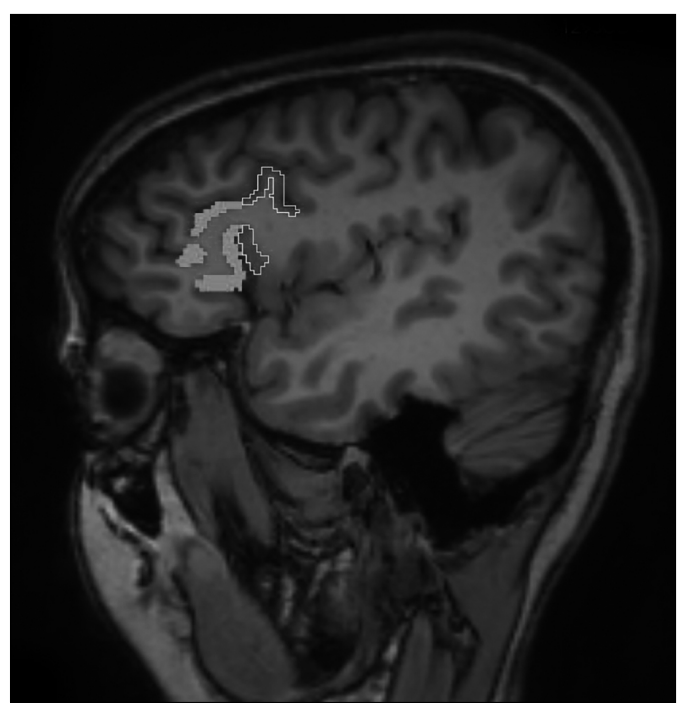

Fig. 1. Tracing of pars traingularis (thick tracing) and pars opercularis (thin tracing) in one of the sagittal sections of brain. cus, inferiorly by the AAR and caudally by the pre-central sulcus. Each region was measured independently and in both the left and right hemispheres of each subject. Inter-rater reliability was established by two investigators measuring the Rol in a sample of randomly selected images. Inter-rater reliability was $>80 \%$ and average intra-class correlation being $>0.9$.

\section{Statistical Analyses}

The normality of the data was examined using the Kolmogorov - Smirnov test, following which parametric statistical analyses were applied. The socio-demographic data were analyzed using the Independent samples $t$ test and the chi-square test. The effect of the Schneiderian First Rank Symptoms status (i.e., FRS[+], FRS[-], and HC) was examined using the Multivariate Analyses of Covariance (MANCOVA) controlling for potential confounding effects of age, sex, and intracranial volume. Post-hoc pairwise comparison was conducted with $p$ value adjusted for multiple comparison correction by the Bonferroni method. The asymmetry index of regional volumes was calculated as volumes of $\{$ (Left volume - Right volume $) \times 100 /$ (Left volume + Right volume) $\}$, with positive values suggesting leftward asymmetry and negative values indicating rightward asymmetry. The statistical significance was set at $p<0.05$ (two-tailed).

\section{RESULTS}

The age (years) and gender distribution among the three groups did not differ significantly. The FRS(+) patients had later age at onset with more positive symptoms compared to FRS ( - ) patients who had higher negative symptoms and longer duration of untreated illness (Table 1). On Pearson's correlation, no significant association was noted between any of these clinical variables with PTr \& POp volumetric/asymmetric measures.

MANCOVA test showed FRS status to have a significant effect on the volume of the right PTr and PTr asymmetry but not on the left PTr and POp volumes/asymmetry (Wilks' $\lambda=0.74, \mathrm{~F}=2.41, p<0.01$, partial $\eta^{2}=0.14$ ) (Table 2). Follow-up post hoc analyses revealed FRS + ) patients to have significant deficit in right PTr in comparison with $\mathrm{HC}$ ( $p_{\text {corrected }}=0.03$ ); however, $\mathrm{FRS}(-)$ patients did not differ from $\mathrm{HC}\left(p_{\text {corrected }}=0.18\right)$ as well as FRS $(+)$ $\left(p_{\text {corrected }}=1\right)$. There was no significant effect of sex of the 
Table 1. Demographic and clinical description of FRS(+), FRS(-) and healthy controls

\begin{tabular}{lccccc}
\hline \multicolumn{1}{c}{ Characteristics } & FRS $(+)(\mathrm{n}=27)$ & FRS $(-)(\mathrm{n}=24)$ & Controls $(\mathrm{n}=52)$ & T/ $\chi^{2}$ & $p$ value \\
\hline Age $(\mathrm{yr})$ & $30.56 \pm 7.61$ & $27.58 \pm 5.63$ & $27.23 \pm 7.35$ & 2.08 & 0.13 \\
Sex, M:F & $12: 15$ & $13: 11$ & $26: 26$ & 0.49 & 0.78 \\
Age at onset in years & $28.01 \pm 7.50$ & $24.17 \pm 5.30$ & - & 2.07 & 0.04 \\
Duration of untreated illness (mo) & $19.27 \pm 24.54$ & $40.50 \pm 33.66$ & - & 2.56 & 0.01 \\
Total SAPS & $42.32 \pm 8.60$ & $23.58 \pm 12.44$ & - & 6.15 & $<0.01$ \\
Total SANS & $45.20 \pm 23.71$ & $75.75 \pm 25.66$ & - & 4.33 & $<0.01$ \\
\hline
\end{tabular}

Values are presented as number only or mean \pm standard deviation.

FRS(+), schizophrenia with first rank symptoms; FRS(-), schizophrenia without first rank symptoms; SAPS, scale for the assessment of positive symptoms; SANS, scale for the assessment of negative symptoms.

Table 2. Cortical volume and asymmetry indices of IFG sub-regions in FRS(+), FRS( - ) patients and healthy controls

\begin{tabular}{lcccccc}
\hline \multicolumn{1}{c}{ Brain region } & $\mathrm{FRS}(+)(\mathrm{n}=27)$ & $\mathrm{FRS}(-)(\mathrm{n}=24)$ & Controls $(\mathrm{n}=52)$ & $\mathrm{F}$ & $p$ value & Partial $\eta^{2}$ \\
\hline Right pars triangularis & $1.74 \pm 0.5$ & $1.9 \pm 0.5$ & $2.16 \pm 0.69$ & 4.12 & 0.02 & 0.08 \\
Left pars triangularis & $2.16 \pm 0.63$ & $2.12 \pm 0.82$ & $2.1 \pm 0.57$ & 0.21 & 0.81 & $<0.01$ \\
Right pars opercularis & $1.7 \pm 0.54$ & $1.65 \pm 0.44$ & $1.73 \pm 0.51$ & 0.08 & 0.92 & $<0.01$ \\
Left pars opercularis & $1.78 \pm 0.4$ & $1.6 \pm 0.5$ & $1.74 \pm 0.52$ & 1.41 & 0.25 & 0.03 \\
Asymmetry index pars triangularis & $10.82 \pm 15.62$ & $3.46 \pm 19.49$ & $-0.47 \pm 16.25$ & 3.61 & 0.03 & 0.07 \\
Asymmetry index pars opercularis & $3.02 \pm 13.96$ & $-1.9 \pm 17.27$ & $0.29 \pm 15.87$ & 1.33 & 0.27 & 0.03 \\
\hline
\end{tabular}

Values are presented as mean \pm standard deviation.

FRS(+), schizophrenia with first rank symptoms; FRS(-), schizophrenia without first rank symptoms; IFG, inferior frontal gyrus.

subject on the right PTr volume in this analysis. There was a significant leftward asymmetry of PTr in patients with FRS in contrast to HC (pcorrected $=0.03$ ). Since there were significant differences between FRS $(+)$ and FRS $(-)$ patients in age at onset, duration of untreated illness and negative symptoms, we examined for potential effect of any of these three variables on the PTr asymmetry in patients. These three variables did not have any significant effect on the PTr asymmetry in comparative analyses between FRS $(+)$ and FRS $(-)$ patients (age at onset $[\mathrm{F}=$ $0.01 ; p=0.93]$; duration of untreated illness $[\mathrm{F}=0.01 ; p=$ $0.93]$ and negative symptoms $[\mathrm{F}=1.28 ; p=0.26]$ ). Besides, none of these three variables had any significant correlation with PTr asymmetry. Pearson's correlation test did not show any significant relationship between PTr asymmetry and age at onset $(r=0.04 ; p=0.78)$, duration of untreated illness $(r=-0.1 ; p=0.48)$ and negative symptoms $(r=0.05 ; p=0.74)$.

\section{DISCUSSION}

The findings of this study provide a possible clue for the neural basis of language dysfunction in schizophrenia, i.e., the cortical volume loss in the right PTr. The Right PTr comprises the homologous Broca's area. The phonological aspect of speech, consisting of both sensory and motor facets, is located in the left hemisphere. The role of the right hemisphere in language functions, in contrast to the left hemisphere, is not associated with phonology or syntax. The secondary lexico-semantic storehouse of speech, which provides meaning at the level of sentence and discourse, is located in the right hemisphere. This brain region is responsible for higher-order language functions, which are necessary for the proper understanding and interpretation of someone's real communicative intent [16]. PTr, the motor engrams of the right side, would assimilate the generated meanings from the receptive fields and generate thoughts and intentions with consequent speech and actions. The disintegration of these language components would result in loss of boundaries between thought, speech, and action manifesting as FRS like passivity phenomenon and auditory hallucinations [16,17].

Though the loss of asymmetry, non-right handedness, and right lateralization of language areas like planum temporale has been a consistent feature in schizophrenia $[12,28]$; the exaggerated leftward asymmetry of the PTr seems to define the first-rank symptoms. This may seem to contradict the reduced functional lateralization (reduced 
left lateralization) of language in schizophrenia $[29,30]$. But, the differences in cellular architecture, connectivity, and language functioning of IFG across the hemispheres leads to dissociation of functional lateralization and structural asymmetry [15]. The morphological asymmetry of Broca's region is not reproducibly demonstrated in healthy human subjects and noted to be mostly symmetrical with a wide variance, as observed in this study [15].

The exaggerated leftward asymmetry is a consequence of a higher volume deficit on the right side rather than an increased left PTr volume. This anatomical deficit is supported by the findings of the loss of "Yakovlevian torque" (i.e., reversal/ loss of the "right frontal petalia being greater than left") in schizophrenia $[1,31]$. In the human brain, the right frontal region tends to warp slightly forward relative to left and twists around Sylvain fissure termed Yakovlevian torque. Evolutionarily, this frontal protrusion signifies the expansion of the prefrontal cortex with the advancement of speech [32]. The torque forms one of the bases of asymmetry of the brain closely associated with language development [1]. Exaggeration of leftward asymmetry in PTr of schizophrenia suggests abrogation of its role on the right side, resulting in First rank symptoms.

Interestingly, IFG, including PTr, plays a significant role in the mirror neuron system (MNS) for proper understanding and interpretation of the action of others [33]. Comprehension of symbolic language is evolved from understanding the symbolic manual gestures of others, and both are neurobiologically interknit in IFG [34]. Dysfunctional MNS with impaired social cognition is well studied in schizophrenia and would describe the role of PTr in the pathogenesis [35]. The patients with schizophrenia without FRS had prominent negative symptoms but showed a paradoxical absence [36] of a significant difference in volume and asymmetry indices of PTr. This further suggests the specificity of the right PTr in the genesis of FRS.

Several studies have reported right PTr cortical volume loss in schizophrenia [37-40]. To the best of our knowledge, this is the first-time manual morphometry study looking into the PTr volume deficits in antipsychotic-naïve schizophrenia patients with FRS. The antipsychotic-naïve status rules out the possible confounding effects of antipsychotics; examination of early course schizophrenia patients avoided the potential confounding influence of illness chronicity.

Nonetheless, we wish to mention that since the schizo- phrenia patients were examined during the acute phase of the illness prior to antipsychotic treatment, it is possible that some patients might not have reported their symptoms such as hallucination and delusion. While independent assessment by two clinicians would have reduced this possibility, the likelihood of potential falsenegative ascertainment for FRS cannot be ruled out completely. We acknowledge this as a potential limitation; in future studies, repeat assessment for FRS after short-term treatment will add to the methodological rigour of the study.

In summary, this study observation suggests reduced right PTr volume with leftward asymmetry to be associated with FRS in schizophrenia. This is consistent with the loss of Yakovlevian torque in schizophrenia. The volume and asymmetry correlate of PTr with indices of language dysfunction and its specific role in the pathogenesis of various symptoms of schizophrenia, especially self-disorder and social cognition deficits, needs further evaluation. Given that PTr contains mirror neurons, such research studies can help in understanding the relationship between dysfunctional mirror neuron system and the genesis of psychiatric disorders like schizophrenia [41].

\section{Acknowledgments}

VS is supported by Department of Biotechnology (DBT) Wellcome Trust India Alliance (IA/CPHE/18/1/503956). VSS is supported by Department of Biotechnology (DBT) Wellcome Trust India Alliance (IA/CRC/19/1/610005). GV acknowledges the support of the Department of Biotechnology, Government of India (BT/HRD-NBA-NWB/ 38/2019-20(6)) grant to GV.

GV acknowledges the support of the SwarnaJayanti Fellowship by the Department of Science and Technology, Government of India (DST/SJF/LSA-02/2014-15).

\section{Conflicts of Interest}

No potential conflict of interest relevant to this article was reported.

\section{Author Contributions}

Conceptualization: Ganesan Venkatasubramanian, Bangalore Nanjundaiah Gangadhar. Data acquisition: Venkataram Shivakumar, Sunil Vasu Kalmady, Ganesan Venkatasubramanian. Data analysis: Venkataram Shivakumar, Vanteemar Sathyanarayana Sreeraj, Sunil Vasu Kalmady. 
Acquired the funding: Ganesan Venkatasubramanian. Writing - original draft: Venkataram Shivakumar, Vanteemar Sathyanarayana Sreeraj, Ganesan Venkatasubramanian. All authors reviewed and contributed to the final draft.

\section{ORCID}

Venkataram Shivakumar

https://orcid.org/0000-0003-0353-8597

Vanteemar Sathyanarayana Sreeraj

https://orcid.org/0000-0002-2946-1228

Sunil Vasu Kalmady

https://orcid.org/0000-0002-4876-9121

Bangalore Nanjundaiah Gangadhar

https://orcid.org/0000-0002-9887-6515

Ganesan Venkatasubramanian

https://orcid.org/0000-0002-0949-898X

\section{REFERENCES}

1. Crow TJ. Schizophrenia as the price that homo sapiens pays for language: a resolution of the central paradox in the origin of the species. Brain Res Brain Res Rev 2000;31:118-129.

2. Crow TJ. The 'big bang' theory of the origin of psychosis and the faculty of language. Schizophr Res 2008;102:31-52.

3. Nestor PG, Han SD, Niznikiewicz M, Salisbury D, Spencer K, Shenton ME, et al. Semantic disturbance in schizophrenia and its relationship to the cognitive neuroscience of attention. Biol Psychol 2001;57:23-46.

4. Kerns JG, Berenbaum H, Barch DM, Banich MT, Stolar N. Word production in schizophrenia and its relationship to positive symptoms. Psychiatry Res 1999;87:29-37.

5. Condray R, van Kammen DP, Steinhauer SR, Kasparek A, Yao JK. Language comprehension in schizophrenia: trait or state indicator? Biol Psychiatry 1995;38:287-296.

6. Costafreda SG, Fu CH, Lee L, Everitt B, Brammer MJ, David AS. A systematic review and quantitative appraisal of $\mathrm{fMRI}$ studies of verbal fluency: role of the left inferior frontal gyrus. Hum Brain Mapp 2006;27:799-810.

7. Hagoort P. On Broca, brain, and binding: a new framework. Trends Cogn Sci 2005;9:416-423.

8. Suga $\mathrm{M}$, Yamasue $\mathrm{H}$, Abe $\mathrm{O}$, Yamasaki $\mathrm{S}$, Yamada $\mathrm{H}$, Inoue $\mathrm{H}$, et al. Reduced gray matter volume of Brodmann's Area 45 is associated with severe psychotic symptoms in patients with schizophrenia. Eur Arch Psychiatry Clin Neurosci 2010;260: 465-473.

9. Suzuki M, Zhou SY, Takahashi T, Hagino H, Kawasaki Y, Niu $\mathrm{L}$, et al. Differential contributions of prefrontal and temporolimbic pathology to mechanisms of psychosis. Brain 2005; 128(Pt 9):2109-2122.

10. Broca P. Rapport sur un memoire de M. Armand de Fleury intitule: de l'inegalite dynamique des deux hemispheres cerebraux.
Bull Acad Med 1877;6:508-539.

11. Gruzelier JH. Functional neuropsychophysiological asymmetry in schizophrenia: a review and reorientation. Schizophr Bull 1999;25:91-120.

12. Sommer I, Ramsey N, Kahn R, Aleman A, Bouma A. Handedness, language lateralisation and anatomical asymmetry in schizophrenia: meta-analysis. Br J Psychiatry 2001;178:344-351.

13. Sumich A, Chitnis XA, Fannon DG, O'Ceallaigh S, Doku VC, Falrowicz $\mathrm{A}$, et al. Temporal lobe abnormalities in first-episode psychosis. Am J Psychiatry 2002;159:1232-1235.

14. Chou PH, Lin WH, Li WR, Huang CM, Sun CW. Reduced language lateralization in first episode schizophrenia: a near infrared spectroscopy study. Prog Neuropsychopharmacol Biol Psychiatry 2017;78:96-104.

15. Keller SS, Crow T, Foundas A, Amunts K, Roberts N. Broca's area: nomenclature, anatomy, typology and asymmetry. Brain Lang 2009;109:29-48.

16. Mitchell RL, Crow TJ. Right hemisphere language functions and schizophrenia: the forgotten hemisphere? Brain 2005; 128(Pt 5):963-978.

17. Crow TJ. Handedness, language lateralisation and anatomical asymmetry: relevance of protocadherin XY to hominid speciation and the aetiology of psychosis. Point of view. BrJ Psychiatry 2002;181:295-297.

18. Toh WL, Castle DJ, Rossell SL. What is the future for Schneiderian first-rank symptoms, in the Diagnostic and Statistical Manual of Mental Disorders and otherwise? Aust N ZJ Psychiatry 2016;50:831-833.

19. Rao NP, Venkatasubramanian G, Arasappa R, Gangadhar BN. Relationship between corpus callosum abnormalities and schneiderian first-rank symptoms in antipsychotic-naive schizophrenia patients. I Neuropsychiatry Clin Neurosci 2011;23:155-162.

20. Heinz A, Voss M, Lawrie SM, Mishara A, Bauer M, Gallinat J, et al. Shall we really say goodbye to first rank symptoms? Eur Psychiatry 2016;37:8-13.

21. Venkatasubramanian G, Arasappa R, Rao NP, Gangadhar BN. Digit ratio (2D:4D) asymmetry and Schneiderian first rank symptoms: implications for cerebral lateralisation theories of schizophrenia. Laterality 2011;16:499-512.

22. Venkatasubramanian G, Jayakumar PN, Keshavan MS, Gangadhar BN. Schneiderian first rank symptoms and inferior parietal lobule cortical thickness in antipsychotic-naïve schizophrenia. Prog Neuropsychopharmacol Biol Psychiatry 2011;35:40-46.

23. Sheehan DV, Lecrubier $\mathrm{Y}$, Sheehan $\mathrm{KH}$, Amorim $\mathrm{P}$, Janavs J, Weiller E, et al. The Mini-International Neuropsychiatric Interview (M.I.N.I.): the development and validation of a structured diagnostic psychiatric interview for DSM-IV and ICD-10. J Clin Psychiatry 1998;59 Supp/ 20:22-33; quiz 34-57.

24. Mellor CS. First rank symptoms of schizophrenia. I. The frequnncy in schizophrenics on admission to hospital. II. Differences between individual first rank symptoms. Br J Psychiatry 
1970;117:15-23.

25. Andreasen NC. Scale for the assessment of negative symptoms (SANS). lowa City:University of lowa; 1983.

26. Andreasen NC. The scale for the assessment of positive symptoms (SAPS). Iowa City:University of lowa;1984.

27. Foundas AL, Eure KF, Luevano LF, Weinberger DR. MR/ asymmetries of Broca's area: the pars triangularis and pars opercularis. Brain Lang 1998;64:282-296.

28. Hirnstein M, Hugdahl K. Excess of non-right-handedness in schizophrenia: meta-analysis of gender effects and potential biases in handedness assessment. Br J Psychiatry 2014;205: 260-267.

29. Ocklenburg S, Westerhausen R, Hirnstein M, Hugdahl K. Auditory hallucinations and reduced language lateralization in schizophrenia: a meta-analysis of dichotic listening studies. J Int Neuropsychol Soc 2013;19:410-418.

30. Tréhout M, Leroux E, Delcroix N, Dollfus S. Relationships between corpus callosum and language lateralization in patients with schizophrenia and bipolar disorders. Bipolar Disord 2017; 19:496-504.

31. Petty RG. Structural asymmetries of the human brain and their disturbance in schizophrenia. Schizophr Bull 1999;25:121-139.

32. Xiang L, Crow T, Roberts N. Cerebral torque is human specific and unrelated to brain size. Brain Struct Funct 2019;224: 1141-1150.

33. Rizzolatti G, Fogassi L, Gallese V. Neurophysiological mechanisms underlying the understanding and imitation of action. Nat Rev Neurosci 2001;2:661-670.

34. Corballis MC. Mirror neurons and the evolution of language. Brain Lang 2010;112:25-35.
35. Maat A, van Haren NEM, Bartholomeusz CF, Kahn RS, Cahn W. Emotion recognition and theory of mind are related to gray matter volume of the prefrontal cortex in schizophrenia. Eur Neuropsychopharmacol 2016;26:255-264.

36. Galderisi S, Merlotti E, Mucci A. Neurobiological background of negative symptoms. Eur Arch Psychiatry Clin Neurosci 2015;265:543-558.

37. Zhang W, Deng W, Yao L, Xiao Y, Li F, Liu J, et al. Brain structural abnormalities in a group of never-medicated patients with long-term schizophrenia. Am / Psychiatry 2015;172: 995-1003.

38. Venkatasubramanian G, Jayakumar PN, Gangadhar BN, Keshavan MS. Automated MRI parcellation study of regional volume and thickness of prefrontal cortex (PFC) in antipsychotic-naïve schizophrenia. Acta Psychiatr Scand 2008; 117:420-431.

39. Bhojraj TS, Francis AN, Rajarethinam R, Eack S, Kulkarni S, Prasad KM, et al. Verbal fluency deficits and altered lateralization of language brain areas in individuals genetically predisposed to schizophrenia. Schizophr Res 2009;115:202-208.

40. Iwashiro N, Suga M, Takano $Y$, Inoue $H$, Natsubori T, Satomura $\mathrm{Y}$, et al. Localized gray matter volume reductions in the pars triangularis of the inferior frontal gyrus in individuals at clinical high-risk for psychosis and first episode for schizophrenia. Schizophr Res 2012;137:124-131.

41. Jeon H, Lee SH. From neurons to social beings: short review of the mirror neuron system research and its socio-psychological and psychiatric implications. Clin Psychopharmacol Neurosci 2018;16:18-31. 